\title{
PACKET SCHEDULING IN THE PRESENCE OF CHANNEL ESTIMATION ERROR IN MULTI-USER OFDM WIRELESS SYSTEMS
}

\author{
Jae-Hoon Jeon and Yong-Hwan Lee \\ School of Electrical Engineering and INMC, Seoul National University \\ Kwanak P. O. Box 34, Seoul, 151-600 Korea \\ e-mail: ylee@snu.ac.kr
}

\begin{abstract}
In this paper, we investigate the performance of a packetbased multi-user orthogonal frequency division multiplexing (OFDM) wireless system in the presence of channel estimation error. To this end, we consider the use of two channel estimation schemes for coherent demodulation; one is a simple linear and the other is an optimum Wiener-type channel estimator. We examine the effect of channel impulse response (CIR) estimation error on the coherent reception and then the effect of signal-tointerference and noise ratio (SINR) estimation error on channel aware transmission techniques (e.g., packet scheduling). It is shown that the performance of coherent reception is relatively less susceptible to the CIR error, but the performance of channel aware schemes can be very sensitive to the SINR estimation error, mainly due to incorrect scheduling. To alleviate this problem, we propose a scheduling scheme which can work without significant performance degradation in the presence of large channel estimation error. Finally, we verify the performance of the proposed scheduling scheme by computer simulation.
\end{abstract}

\section{Introduction}

In recent years, multi-user orthogonal frequency division multiplexing (OFDM) has attracted much attention as an effective transceiver technique for high-speed packetbased multi-user wireless systems. The OFDM can provide a high spectral efficiency by incorporating advanced transmission technologies. It is known that the performance of OFDM systems can significantly be enhanced by employing channel-aware transmission techniques. In fact, opportunistic scheduling techniques with the use of adaptive modulation and coding (AMC) and hybrid automatic repeat request (HARQ) can significantly enhance the average spectral efficiency of packet-based OFDM wireless systems [1].

Most of previous researches assumed the use of perfect channel state information (CSI) (e.g., the channel impulse response (CIR) and signal-to-interference and noise power ratio (SINR)) in the transmitter [2]. However, it may not be practical to obtain accurate CSI in harsh wireless environments. Recently, a few researchers have investigated the effect of channel estimation error on the linkadaptation, AMC and HARQ in single-user systems [2, 3]. However, to the authors' best knowledge, the no result has been reported on the effect of channel estimation error on multi-user systems.

In this paper, we investigate the downlink performance of a packet-based multi-user OFDM wireless system in the presence of channel estimation error, especially in view of packet scheduling and link adaptation. To this end, we consider the use of two estimation schemes; one is a simple linear and the other is an optimum Wiener-type CSI estimator [4, 5]. We first examine the effect of CIR estimation error on the coherent reception and the effect of instantaneous SINR estimation error on the channel aware transmission schemes (e.g., packet scheduling). To alleviate the performance degradation due to channel estimation error, we propose a packet scheduler that allocates the resource to the user in a different manner according to the channel condition. The performance of the proposed scheduler is verified by computer simulation.

\section{System framework}

Consider an OFDM downlink system as depicted in Fig. 1, where $X_{m}(n, k)$ represents the $m$-th user signal at the $n$-th symbol time and the $k$-th subcarrier, $m \in\{0,1$, $\cdots, M-1\}$, and $k \in\{0,1, \cdots, K-1\}$. The frequency domain symbol is converted into a time domain signal using the inverse fast Fourier transform (FFT). A cyclic prefix (CP) is inserted to each frame to preserve the orthogonality between the subcarriers and to eliminate the interference between the adjacent OFDM symbols. We assume that each data packet comprises $N_{t}$ OFDM symbols and $N_{f}$ subcarriers, and that the pilot symbol is regularly inserted in a rectangular shape (i.e., apart by $d_{t}$ and $d_{f}$ symbols in the time and frequency grid, respectively).

Then, the received signal after FFT can be written as 


$$
Y_{m}(n, k)=H_{m}(n, k) X(n, k)+Z_{m}(n, k)
$$

where $X(n, k)$ is the user data signal, $Z_{m}(n, k)$ is the background noise plus interference term which can be approximated as zero mean additive white Gaussian noise (AWGN) with variance $\sigma_{m, Z}^{2}$, and $H_{m}(n, k)$ is the frequency response of the fading channel from the transmitter to the $m$-th user, which is the discrete Fourier transform of the CIR represented as

$$
h_{m}(t, \tau)=\sum_{l=0}^{L-1} h_{m, l}(t) \delta\left(\tau-\tau_{m, l}\right) .
$$

Here, $L$ is the number of multipaths, $\delta(\cdot)$ denotes Kronecker delta function, $\tau_{m, l}$ and $h_{m, l}(t)$ are the delay and complex-valued CIR of the $l$-th path of user $m$ at time $t$, respectively.

In the receiver, all the users estimate the CSI and report their estimated CSI to the transmitter through a feedback channel in the uplink. Then, the transmitter selects a user to be served at the next packet time. Accordingly, the AMC parameters for the selected user are determined based on the instantaneous CSI. In what follows, we describe a procedure for the scheduling, link-adaptation and channel estimation.

\subsection{Packet scheduling}

It was known that the spectral efficiency can significantly be improved by employing an intelligent packet scheduling scheme taking account of wireless channel condition, so-called the opportunistic packet scheduling [7-10]. The maximum SINR scheduling and proportional fair (PF) scheduling are typical examples of opportunistic packet scheduling.

The maximum SINR scheduling selects a user whose instantaneous SINR is the largest among the users as

$$
m=\underset{m \in\{0, \cdots M-1\}}{\arg } \max \left[\gamma_{m}(n, k)\right]
$$

where $\gamma_{m}(n, k)$ denotes the instantaneous SINR of user $m$. If we assume that $M$ users are uniformly allocated to each subcarrier, the subcarrier index $k$ in (3) can be omitted without loss of generality. This implies that the multi-carrier system with an opportunistic scheduler can be treated as a simple parallel extension of a single-carrier time division multiplexing system [8]. Therefore, we will omit the index $k$ in what follows. The maximum SINR scheduler maximizes the spectral efficiency by achieving the multi-user diversity (MUD) gain. However, it cannot guarantee the fairness if the difference between the average SINR $\bar{\gamma}_{m}$ of each user is large. This fairness problem can be alleviated by employing a PF scheduling scheme [9].

Letting $R_{m}(n)$ be a possible transmission data rate at symbol time $n$ and $\bar{R}_{i}(n)$ be the average data rate by that symbol time $n$, the PF scheduler selects a user according to

$$
m=\underset{m \in\{0, \cdots M-1\}}{\arg } \max \left[\frac{R_{m}(n)}{\bar{R}_{m}(n)}\right] .
$$

Then, (4) can be rewritten as [10]

$$
\begin{aligned}
m & =\underset{m \in\{0, \cdots M-1\}}{\arg } \max \left[\frac{\gamma_{m}(n)}{\bar{\gamma}_{m}}\right] \\
& =\underset{m \in\{0, \cdots M-1\}}{\arg \max }\left[\left|H_{m}(n)\right|^{2}\right] .
\end{aligned}
$$

In this paper, we consider the use of PF scheduling considering the trade-off issues between the spectral efficiency and the fairness.

\subsection{AMC and HARQ}

The AMC adjusts the modulation level and code rate according to the channel status, requiring accurate CSI such as the instantaneous SINR. Thus, the performance of AMC can significantly be degraded in the presence of channel estimation error. This problem can be alleviated with combined use of HARQ that can support the rate adaptation by retransmission.

The HARQ combines the channel coding and ARQ to make the retransmissions more reliable [11]. It often employs a stop-and-wait method to save the radio resource. The HARQ with chase combining (CC) repeatedly sends a copy of originally transmitted packet when a decoding failure occurs. The receiver softly combines the received packet and previously received packets in a dynamic manner. The HARQ with incremental redundancy (IR) incrementally sends an additional redundant data when the transmission fails. Thus, the HARQ-IR needs ratecompatible codes supported by a simple puncturing operation. It is known that the HARQ-IR can provide better performance than the HARQ-CC due to an additional coding gain at the cost of increased decoding complexity.

The effective code rate is adjusted according to the channel status with an acknowledge signaling (e.g., ACK or NACK). Since the HARQ does not require precise channel information at the transmitter, it can provide performance robust to the channel measurement error. In this paper, we consider the use of an AMC scheme with fixed power constraint, square QAM and Zig-zag coding [12]. We also consider the use of an HARQ-IR scheme to accommodate inaccurate AMC operation. The operation parameters for these techniques are summarized in Table 1.

\subsection{Channel estimation}

Accurate CSI is indispensable for the implementation of channel aware transmission techniques. The CSI can be estimated using the received pilot symbols. The CIR at the pilot symbol time for user $m$ can be estimated as 


$$
\begin{aligned}
\tilde{H}_{m}\left(n_{p}, k_{p}\right) & =Y_{m}\left(n_{p}, k_{p}\right) / X\left(n_{p}, k_{p}\right) \\
& =H_{m}\left(n_{p}, k_{p}\right)+\tilde{Z}_{m}\left(n_{p}, k_{p}\right)
\end{aligned}
$$

where $n_{p}$ and $k_{p}$ denote the symbol and subcarrier index of the pilot symbol, respectively, and $\tilde{Z}_{i}\left(n_{p}, k_{p}\right)$ denotes the additive noise term. The CIR at the data symbol time can be estimated by interpolating the CIR (6) obtained from the pilot symbol.

For the purpose of interpolation, we consider the use of two interpolation filters, Wiener and linear interpolation filter as the channel estimation filter (CEF) [4, 5]. The Wiener CEF minimizes the mean square error (MSE) of the estimated CIR at the cost of large implementation complexity, while the liner interpolation CEF can be implemented with ease at the cost of performance degradation.

The Wiener CEF estimate the CIR at the $n$-th symbol time and the $k$-th subcarrier by

$$
\hat{H}(n, k)=\sum_{p=0}^{N_{w}-1} w_{p}(n, k) \tilde{H}\left(n_{p}, k_{p}\right)
$$

where $N_{w}$ denotes the number of filter taps and $w_{p}(n, k)$ denotes the $p$-th filter-tap coefficient. The optimum coefficient of the Wiener CEF can be determined as [5]

$$
\mathbf{w}^{T}(n, k)=\boldsymbol{\theta}^{T}(n, k) \boldsymbol{\Phi}^{-1}
$$

where the superscript $T$ denotes the matrix transpose, $\boldsymbol{\Phi}$ is the $\left(N_{w} \times N_{w}\right)$ auto-covariance matrix of the received pilot symbol and $\boldsymbol{\theta}$ is the $\left(N_{w} \times 1\right)$ cross-covariance vector between the desired signal and received pilot symbol.

In the linear interpolation CEF, the CIR at the data symbol is estimated as [4]

$$
\begin{aligned}
& \hat{H}\left(n_{p}, k_{p}+k\right)=\tilde{H}\left(n_{p}, k_{p}\right)+\frac{k}{d_{f}}\left(\tilde{H}\left(n_{p}, k_{p}+d_{f}\right)-\tilde{H}\left(n_{p}, k_{p}\right)\right), 0<k<d_{f} \\
& \hat{H}\left(n_{p}+n, k\right)=\tilde{H}\left(n_{p}, k\right)+\frac{n}{d_{t}}\left(\tilde{H}\left(n_{p}+d_{t}, k\right)-\tilde{H}\left(n_{p}, k\right)\right), 0<n<d_{t} .
\end{aligned}
$$

The channel SINR can be estimated using the estimated CIR. Since the average SINR can be estimated by a long term average with a relatively high accuracy [6], we assume that the average SINR $\bar{\gamma}_{m}$ can be estimated very accurately. Then, the instantaneous SINR can be estimated as

$$
\hat{\gamma}_{m}(n, k)=\left|\hat{H}_{m}(n, k)\right|^{2} \bar{\gamma}_{m}
$$

\section{Effect of channel estimation error}

Accurate CSI cannot be estimated due to imperfect channel condition, such as the interference, background noise and channel fading. Inaccurate CSI degrades the system performance in two aspects. The CIR estimation error affects the coherent reception and the instantaneous
SINR estimation error affects the user scheduling and the choice of AMC parameters.

We first examine the effect of CIR estimation error on the coherent reception. Fig. 2 depicts the average spectral efficiency with the use of perfect scheduling and AMC selection, assuming that all the users experience the same Doppler frequency and delay spread, and that all the users are uniformly distributed in a cell. The legends 'LI' and 'ISINR' denote the linear interpolation and instantaneous SINR, respectively. For reference, the performance with ideal channel estimation is also depicted. The simulation condition is summarized in Table 2. It can be seen that the average spectral efficiency improves as the number of user increases by an order of $\log (\log N)$ due to the MUD gain [8]. It can also be seen that the coherent detection is not significantly affected by the CIR estimation error in a packet-based multi-user OFDM system.

Next, we investigate the effect of erroneous SINR on the scheduling and AMC. Fig. 3 depicts the average spectral efficiency of the PF scheduling and AMC assuming perfect coherent detection. It can be seen that the system performance is significantly affected by the SINR estimation error. Unless the SINR is appropriately estimated, the performance of the packet scheduler is severely degraded. This is mainly due to the fact that the scheduler selects an improper user and the AMC level is also incorrectly selected because of inaccurate SINR information. As a result, the actual transmission rate can be much lower than the anticipated one with the use of a simple linear channel estimator. It can also be seen that the performance due to erroneous scheduling is not noticeably affected by the AMC performance. This implies that the performance degradation due to inaccurate AMC selection is significantly mitigated by the HARQ-IR. Therefore, it can be claimed that the performance degradation is mainly due to incorrect scheduling. In the presence of incorrect SINR information, users in poor condition can frequently be selected as the best user, which prevents users in good condition from transmitting the data at a high rate. Thus, the PF scheduler may not guarantee the proportional fairness.

Fig. 4 depicts the probability of incorrect scheduling in terms of the number of users. It can be seen that the performance degradation is getting worse as the number of users increases, especially when a simple linear estimator is employed.

\section{PF scheduling robust to channel information error}

Although the Wiener-type CEF can provide acceptable performance, it may not easily be employed due to the implementation complexity [5]. This problem can be alleviated by employing a scheduling scheme robust to incorrect CSI.

We consider a scheduling scheme that classifies the users 
into several groups based on the accuracy of estimated CSI. Fig. 5 illustrates a block diagram of the proposed scheduler scheme. The proposed scheme first monitors the average CSI of all users in the cell and then classifies the users into plural groups according to the condition of channel estimation accuracy. Assuming that all the users experience the same Doppler shift and delay spread, the average SINR is the only factor affecting the channel estimation accuracy. Thus, we can classify the user condition in terms of the average SINR.

The channel condition of the user can be classified into $I$ numbers of groups according to the average SINR as

$$
U_{i+1} \in m\left(\breve{\gamma}_{i} \leq \bar{\gamma}_{m}<\bar{\gamma}_{i+1}\right), i=0,1, \cdots, I-1
$$

where $U_{i}$ denotes the $i$-th user group and $\breve{\gamma}_{0}(=-\infty)<\breve{\gamma}_{1}<\breve{\gamma}_{2} \cdots<\breve{\gamma}_{I-1}<\breve{\gamma}_{I}(=\infty)$ are the thresholds for the classification. For example, in the case of twogroup classification, the value of threshold $\breve{\gamma}_{1}$ can be determined as the median value of the users' average SINR.

Then, each user group can employ different scheduling algorithms to reduce the probability of incorrect user selection. Finally, the selected user signal in each group is transmitted alternatively. Assuming that the all the users are uniformly distributed in the cell, the proposed PF scheduler can provide an asymptotically proportional fairness even in the presence of large channel estimation error.

Fig. 6 depicts the performance of the proposed scheduler with $I=2$, assuming that the average SINR of users is uniformly distributed between -10 and $30 \mathrm{~dB}$, and $\breve{\gamma}_{1}=10 \mathrm{~dB}$. The users are classified into two groups: One group contains users in good channel condition and the other group contains users in poor condition. The users in good condition are scheduled using a PF scheduler, while the users in poor condition are scheduled using a nonopportunistic scheduler (e.g., round-robin type). It can be seen that the proposed scheduler provides performance quite comparable to the use of Wiener-type estimator in the presence of the channel estimation error (i.e., with the use of a simple linear interpolator). Thus, the proposed scheduling scheme can be combined with the use of a simple channel estimator, substantially reducing the implementation complexity for the channel estimation. Note that similar approaches can also be applied to the cases when the users experience different Doppler shifts and delay spreads.

\section{Conclusions}

In this paper, we have investigated the effect of channel estimation error in a packet-based multi-user OFDM system. The simulation results show that the CIR estimation error is not critical for the coherent reception, but the SINR estimation error is critical for the use of channel-aware transmission techniques such as the packet scheduling and AMC. We have proposed a scheduling scheme to mitigate the effect of channel estimation error. The proposed scheme classifies the users into a number of groups according to the accuracy of CSI and employs different scheduling policies for each group. The simulation results show that the proposed scheme can work without noticeable performance degradation in the presence of large channel estimation error, enabling the use of a simple channel estimator.

\section{References}

[1] S. Abeta, H. Atarashi and M. Sawahashi, "Broadband packet wireless access incorporating high-speed IP packet transmission,” in Proc. PIMRC, pp. 844-848, Sept. 2002.

[2] A. Czylwik, "Adaptive OFDM for wideband radio channels,” in Proc. IEEE Globecom., vol. 1, pp.18-22, Nov. 1996.

[3] A. J. Goldsmith and S. G. Chua, "Variable-rate variable-power MQAM for fading channels," Proc. IEEE Trans. Commum., Vol. 45, pp.1218-1230, Oct. 1997.

[4] Y. Zhao and A. Huang, "A novel channel estimation method for OFDM mobile communication systems based on pilot signals and transform-domain processing," in Proc. IEEE VTC, Vol.3, pp.2089-2093, May 1997.

[5] P. Hoeher, S. Kaiserand and P. Robertson, "Twodimensional pilot-symbol-aided channel estimation by Wiener filtering,” in Proc. IEEE ICASSP, Vol. 3, pp.21-24, Apr. 1997.

[6] J.-W. Choi, Design of adaptive OFDM wireless transceivers, Ph. D. dissertation, Seoul National University, Aug. 2004.

[7] X. Liu, E. K. P. Chong and N. B. Shroff, "A Framework for Opportunistic Scheduling in Wireless Networks," Computer Networks, vol. 41, pp. 451-474, Mar. 2003.

[8] P. Svedman, S.K. Wilson, L.J. Cimini and B. Ottersten, "A simplified opportunistic feedback and scheduling scheme for OFDM," in Proc. IEEE VTC, vol. 4, pp.18781882, May 2004.

[9] J. Holtzman, "Asymptotic analysis of proportional fair algorithm," in Proc. IEEE PIMRC, vol. 2, pp. F33-F37, Sept. 2001.

[10] F. Berggren and R. Jannit, "Asymptotically fair scheduling on fading channels," in Proc. IEEE VTC, vol. 4, pp.1934-1938, Sept. 2002.

[11] 3GPP TR25.848, Physical layer aspects of UTRA High Speed Downlink Packet Access, V4.0.0, Mar. 2001.

[12] S. N. Hong and D. J. Shin, "Design of irregular concatenated zigzag codes," in Proc. ISIT, 2005.

[13] W. Rhee and J. M. Cioffi, "Increase in capacity of multiuser OFDM system using dynamic subchannel allocation," in Proc. of IEEE VTC, pp.1085-1089, May 2000. 
Table 1. Operation parameters for AMC and HAQR

\begin{tabular}{|c|c|c|c|c|c|}
\hline \multirow{2}{*}{ AMC set } & \multirow{2}{*}{ Modulation } & \multirow{2}{*}{ Code rate } & Size & \multicolumn{2}{|c|}{ HARQ-IR } \\
\cline { 5 - 6 } & & & (bit/packet) & Retrans. interval & Max. \# of retrans. \\
\hline 1 & QPSK & $3 / 8$ & 384 & 2 & 4 \\
\hline 2 & QPSK & $3 / 4$ & 768 & 2 & 4 \\
\hline 3 & $16 \mathrm{QAM}$ & $9 / 16$ & 1152 & 2 & 4 \\
\hline 4 & $16 \mathrm{QAM}$ & $3 / 4$ & 1536 & 2 & 4 \\
\hline 5 & $64 \mathrm{QAM}$ & $5 / 8$ & 1920 & 2 & 4 \\
\hline 6 & $64 \mathrm{QAM}$ & $3 / 4$ & 2304 & 2 & 4 \\
\hline
\end{tabular}

Table 2. System parameters

\begin{tabular}{|c|c|c|c|}
\hline Index & Value & Index & Value \\
\hline Carrier freq. & $5.8 \mathrm{GHz}$ & Number of sub-carriers & 2048 \\
\hline Cell radius & $2.5 \mathrm{~km}$ & Packet size $\left(N_{t}, N_{f}\right)$ & $(64,8)$ \\
\hline Guard interval & ${ }^{5} \mu^{\mathrm{sec}}$ & Pilot spacing $\left(d_{t}, d_{f}\right)$ & $(64,4)$ \\
\hline Symbol duration $\left(T_{s}\right)$ & $20.48 \mu \mathrm{sec}$ & Pilot pattern & Rectangular \\
\hline Bandwidth & $100 \mathrm{MHz}$ & Doppler effect & $\begin{array}{c}\text { Jakes model } \\
\text { maximum } 16 \mathrm{~Hz}\end{array}$ \\
\hline Duplex & FDD & $\begin{array}{c}\text { Power delay profile } \\
\text { (exponential attenuation) }\end{array}$ & $\begin{array}{c}L=18 \\
\text { rms delay } 167 n \mathrm{sec}\end{array}$ \\
\hline
\end{tabular}

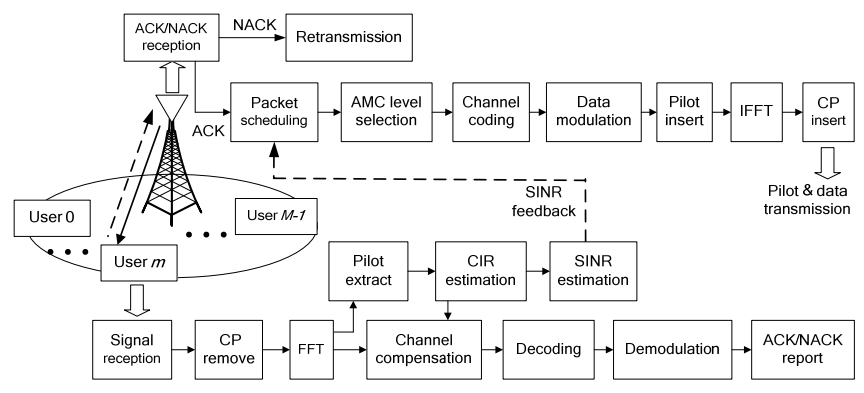

Fig. 1. OFDM structure in consideration.

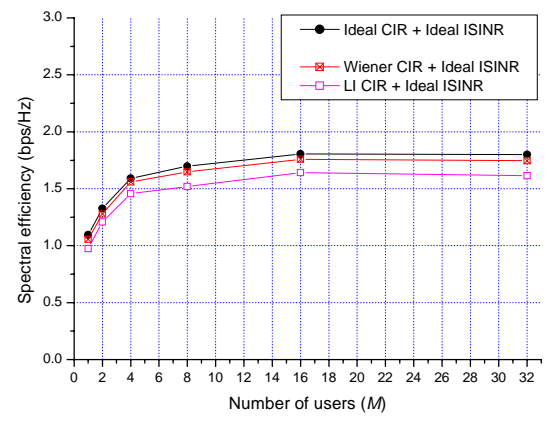

Fig. 2. Average spectral efficiency with imperfect coherent detection.

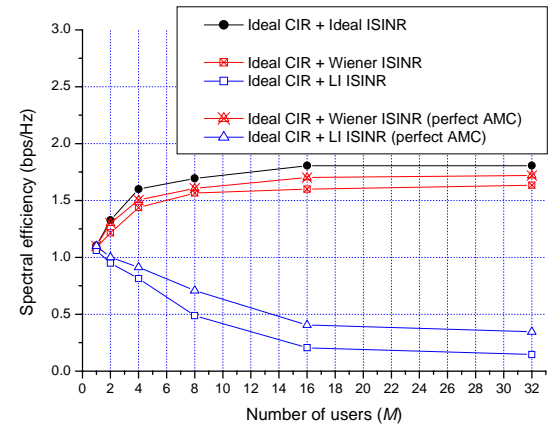

Fig. 3. Average spectral efficiency with imperfect SINR.

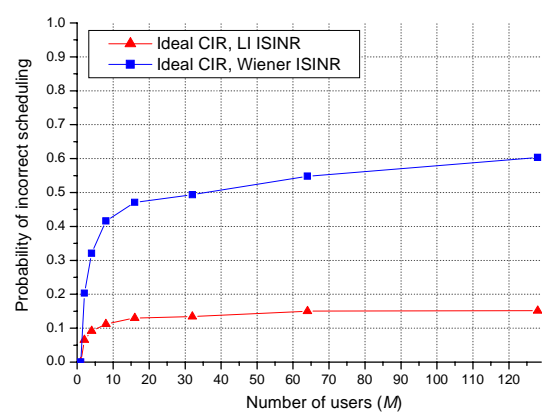

Fig. 4. Probability of incorrect scheduling due to imperfect SINR.



Fig. 5. Structure of the proposed scheduling scheme.

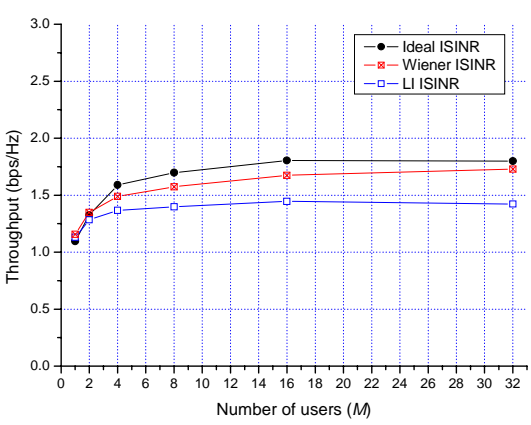

Fig. 6. Performance of the proposed scheduling method. 